\title{
Intermédialités
}

Histoire et théorie des arts, des lettres et des techniques

Intermediality

History and Theory of the Arts, Literature and Technologies

\section{Les inventions de Tendre}

\section{Delphine Denis}

Numéro 4, automne 2004

Aimer

Loving

URI : https://id.erudit.org/iderudit/1005476ar

DOI : https://doi.org/10.7202/1005476ar

Aller au sommaire du numéro

Éditeur(s)

Centre de recherche sur l'intermédialité

ISSN

1705-8546 (imprimé)

1920-3136 (numérique)

Découvrir la revue

Citer cet article

Denis, D. (2004). Les inventions de Tendre. Intermédialités / Intermediality, (4), 44-66. https://doi.org/10.7202/1005476ar
Résumé de l'article

$\mathrm{Du} \mathrm{XVI}^{\mathrm{e}}$ au XVIII ${ }^{\mathrm{e}}$ siècle, l'émergence progressive de la notion d'intimité rend pensable une conception nouvelle de la relation interpersonnelle, dont le XVII ${ }^{\mathrm{e}}$ siècle sut prendre toute la mesure. Parmi les nombreux discours sur l'amour et l'amitié qui accompagnèrent l'institution d'une littérature moderne, le modèle de Tendre, imaginé par Madeleine de Scudéry dans les années 1650, se présente comme un espace de médiations multiples, investi par divers publics, actualisé dans différents supports matériels. En se penchant sur les récits d'invention qui en orchestrèrent l'élaboration, ainsi que sur les modulations esthétiques qu'il suscita, cet article voudrait en dégager les ambiguités et les tensions internes : fragilité indéniable du Royaume de Tendre, mais qui fait précisément toute la richesse de ses propositions.
Ce document est protégé par la loi sur le droit d'auteur. L'utilisation des services d’Érudit (y compris la reproduction) est assujettie à sa politique d'utilisation que vous pouvez consulter en ligne.

https://apropos.erudit.org/fr/usagers/politique-dutilisation/ 


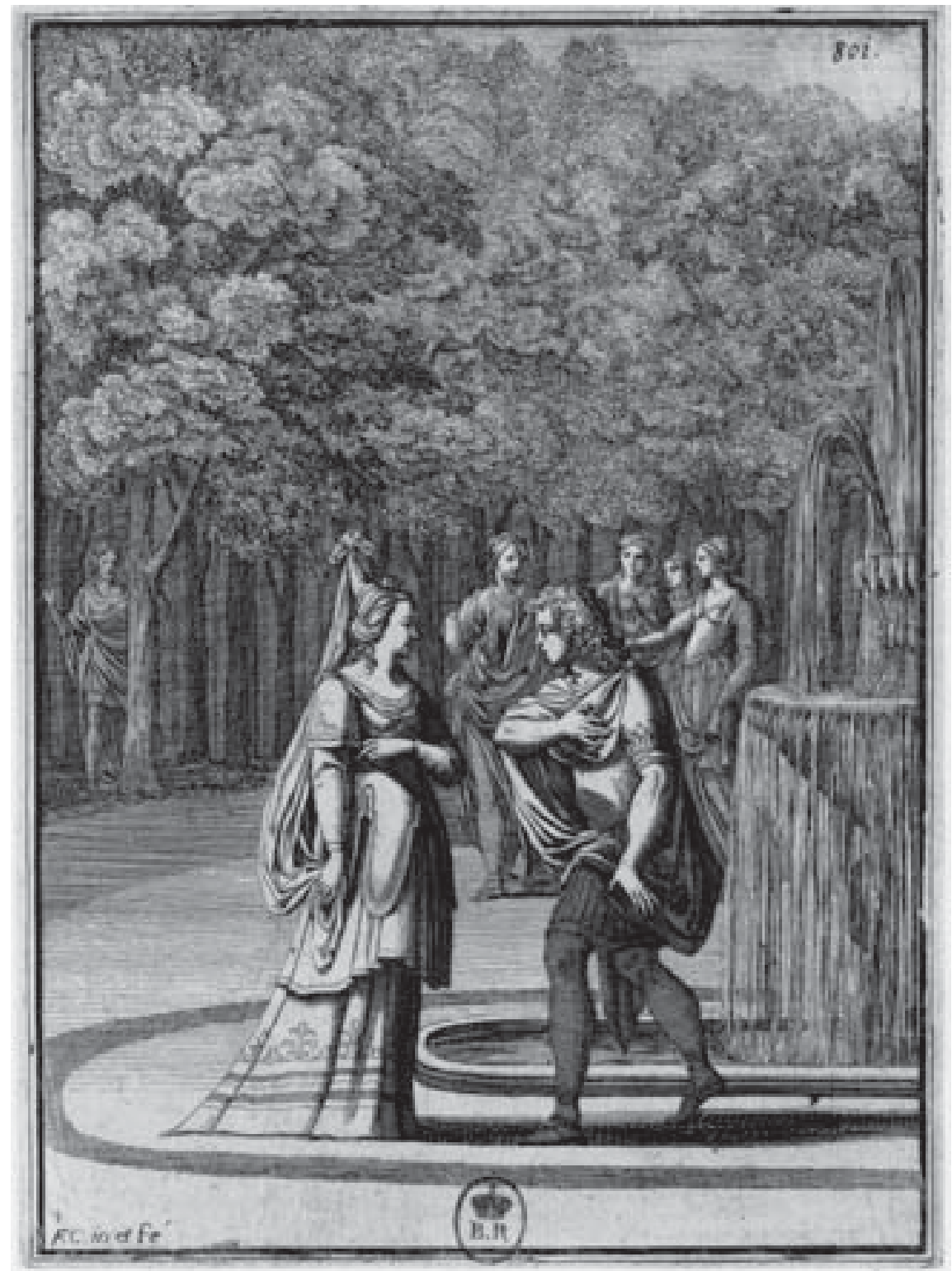

Fig. 1. François Chauveau, Histoire d'Aglatidas et d'Amestris, dans Georges et Madeleine de Scudéry, Artamène ou Le Grand Cyrus, première partie, 1649, coll. Bibliothèque nationale de France. 


\title{
Les inventions de Tendre
}

\author{
Delphine Denis
}

L 'efflorescence de cartographies galantes - bagatelles avouables ou pochades satiriques - qui accompagne au milieu du XVII ${ }^{\mathrm{e}}$ siècle l'exploration de territoires sentis comme inédits n'obéit pas au seul effet de mode, certes indéniable ${ }^{1}$. La représentation spatiale, qu'elle se matérialise en gravures ou organise la logique implicite des discours, relève manifestement d'un souci de repérer, c'est-à-dire aussi de nommer, de nouvelles formes de sociabilité au sein desquelles se rejoue la difficile question des rapports entre hommes et femmes. Sécession précieuse, économie libertine des corps à l'encan, essais d'une politique des cœurs à accorder, les voies sont aussi diverses qu'inégalement frayées. Il y a là, souvent noté, le symptôme de l'inquiétude d'un siècle qui s'interroge sur la validité des anciens modèles du discours amoureux, et dont les propositions conflictuelles cherchent moins à se stabiliser qu'à confronter leurs frontières, à opposer leurs cadastres.

La littérature galante qui s'institue alors a su faire de cette vaste enquête un lieu de débats publics, ouvert par émulation à toutes les plumes du royaume, et surtout à des lecteurs nouveaux dont les libraires comprirent vite qu'il fallait s'attacher les suffrages en flattant leur goût pour ces colifichets de facture inégale. Que les démêlés du Cour avec l'Esprit ou la Raison, les dialogues de

1. Île d'Érotie, Carte de Tendre, Carte du Royaume d'Amour, Royaume de Coquetterie, Carte de l'Empire des Précieuses, Royaume de Galanterie, Carte du Pays de Braquerie, Pays d'Amour... encore la moisson de ces géographies allégoriques est-elle incomplète: voir Enid P. Mayberry Senter, «Les cartes allégoriques romanesques du XVII ${ }^{\mathrm{e}}$ siècle. Aperçu des gravures créées autour de l'apparition de la Carte de Tendre de la Clélie en 1654", Gazette des beaux-arts, vol. 89, n 1299, avril 1977, p. 133-144 et les analyses de JeanMichel Pelous, Amour précieux, amour galant (1654-1675), Paris, Éditions Klincksieck, 1980, p. 13-34 
l'Amour et de l'Amitié, de la Mode et de la Nature, les questions et maximes d'amour indéfiniment posées sans autre résolution que ce retour incessant d'une méfiance partagée aient pu alors conquérir tous les espaces de publication, du manuscrit à l'imprimé, des recueils collectifs aux CEuvres de tel ou telle, est un autre indice de la généralisation du discours sur l'amour, désormais promu véritable «médium de communication ${ }^{2}$ ». Inventivité créatrice et innovations formelles ne furent pas en reste, qui expérimentèrent tous supports et tous prétextes à écriture: rarement fadaises variées en conscience trouvèrent-elles plus de crédit, conventions poétiques dominées plus de défis à relever, dans une surenchère accablante ou réjouissante - affaire de point de vue... De ce bavardage galant, qui rémunère paradoxalement le silence des corps dans les textes $^{3}$, et semble unifier les discours dans une tonalité commune faite d'ingéniosité, de raillerie légère, d'enjouement et de délicatesse, nombreux sont les observateurs qui se sont légitimement efforcés de dégager des lignes de clivage: l'adhésion apparente à cette esthétique ne réduit pas au silence ceux qui, de l'intérieur même, élaborent des propositions singulières, plus ou moins audacieuses, parfois irréductibles. Michel Jeanneret a tout récemment remis en sa lumière crue la force subversive des partisans de l'Éros rebelle ${ }^{4}$ - au risque peut-être de minorer de plus discrètes résistances à la pudibonderie ambiante 5 .

2. L'analyse en est proposée, en termes sociologiques, dans l'ouvrage de Niklas Luhmann, Amour comme passion. De la codification de l'intimité, trad. Anne-Marie Lionnet, Paris, Éditions Aubier, coll. «Présence et pensée», 1990 [1982]. Pour un essai de synthèse sur cette littérature galante dans la seconde moitié du XvII ${ }^{\mathrm{e}}$ siècle, je me permets de renvoyer à mon Parnasse galant. Institution d'une catégorie littéraire au XVII ${ }^{e}$ siècle, Paris, Éditions Honoré Champion, coll. «Lumière classique », $n^{\circ}$ 32, 2001.

3. Voir Éric Méchoulan, Le corps imprimé. Essai sur le silence en littérature, Montréal, Les Éditions Balzac, coll. «L'univers des discours», 1999.

4. Michel Jeanneret, Éros rebelle. Littérature et dissidence à l'âge classique, Paris, Éditions du Seuil, 2003.

5. Mais il est vrai, pour une «Jouissance» - pourtant élégamment suggérée qu'une rare plume féminine osa alors tenter (en l'occurrence, un sonnet de $\mathrm{M}^{\text {lle }}$ Desjardins, composé en 1657), combien de débauches pornographiques dans les recueils collectifs des années $1620 .$. Parnasses «satyriques», «folastres» ou «gaillards » d'accès évidemment barré aux femmes, et devenu d'ailleurs de plus en plus périlleux après le procès de Théophile de Viau en 1623. Sur les premiers essais littéraires de la future $\mathrm{M}^{\mathrm{me}}$ de Villedieu, voir l'étude de Micheline Cuénin, Roman et société sous Louis XIV: Madame de Villedieu, Paris, A.N.R.T-Champion, 1979, p. 104, et la bibliographie plus 
Jean-Michel Pelous était revenu autrefois, dans un livre provocateur et stimulant, sur l'opposition entre préciosité et galanterie ${ }^{6}$ : lues de près, nuancées voire infirmées, ses thèses auront eu le mérite de préciser les formes et les enjeux du discours mondain sur l'amour. Le Royaume de Tendre s'y révélait bien malaisé à situer, comme de nombreux travaux l'ont depuis confirmé ${ }^{7}$. Formation de compromis ambiguë et fragile ${ }^{8}$, le Tendre s'avéra d'abord, au moment de son invention, un espace de médiations aux risques assumés. Que cette modulation particulière du sentiment ait pu trouver à s'accomplir dans des formes littéraires propres - genres et styles - contribua sans nul doute à en élargir les contours : non certes en direction des Terres inconnues, concédées à d'autres explorateurs, mais du côté d'une expression de l'affectivité, promise un siècle plus tard à un bel avenir.

C'est, témoigne Gilles Ménage, Madeleine de Scudéry qui inventa «l'Amour de Tendresse ${ }^{9}$ »: on en pourrait même très précisément dater l'événement, consigné en sa première version dans le recueil manuscrit des Chroniques du

récente de Nathalie Grande, dans Stratégies de romancières. De Clélie à La Princesse de Clèves (1654-1678), Paris, Éditions Honoré Champion, 1999, p. 473-474.

6. Jean-Michel Pelous, Amour précieux, Amour galant (1654-1675).

7. On en trouvera une riche moisson dans la recension bibliographique de Chantal Morlet-Chantalat, Madeleine de Scudéry, Bibliographie des écrivains français, $\mathrm{n}^{\circ} 10$, ParisRome, Éditions Memini, 1997, p. 81-87. À signaler surtout pour notre propos, l'article de Roger Duchêne, «Mademoiselle de Scudéry, reine de Tendre », dans Les trois Scudéry, Actes du colloque du Havre (1 $1^{\text {er }}-5$ octobre 1991), recueillis par Alain Niderst, Paris, Éditions Klincksieck, 1993, p. 625-632; celui de Myriam Maître, «Sapho, reine de Tendre: entre monarchie absolue et royauté littéraire", dans Madeleine de Scudéry: une femme de lettres au XVII siècle, Actes du colloque international de Paris (28-30 juin 2001), Delphine Denis et Anne-Élisabeth Spica (éd.), Arras, Artois Presses Université, 2002, p. 179-193; l'ouvrage de Joan DeJean, Tender Geographies. Women and the Origins of the Novel in France, New York, Columbia University Press, 1991, p. 71-93; l'étude de Chantal MorletChantalat, La Clélie de Mademoiselle de Scudéry, de l'épopée à la Gazette: un discours féminin de la gloire, Paris, Éditions Honoré Champion, coll. «Lumière classique », 1994, p. $559-565$.

8. Voir notamment Éric Méchoulan, Le corps imprimé, p. 67-114; Myriam Maître, Les Précieuses. Naissance des femmes de lettres en France au XVII siècle, Paris, Éditions Honoré Champion, coll. «Lumière classique», 1999, p. 587-596.

9. Gilles Ménage, Menagiana sive excerpta ex ore Egidii Menagii, Paris, Pierre et Florentin Delaulne, 1693, p. 398. 
Samedi tenues par Paul Pellisson ${ }^{10}$ (fig. 1), puis publié sous l'avatar romanesque qu'on lui connaît, dans la première partie de Clélie parue en $1654^{11}$. L'affirmation du savant ami de Sapho - tel est le nom de plume que la romancière s'était choisi - est cependant tout aussi fondée que peu véridique. Historiens et sociologues du for privé l'ont en effet montré ${ }^{12}$, la progressive émergence de la notion d'intimité sur fond de pratiques de plus en plus différenciées rend peu à peu possible, et dès lors formulable, une conception nouvelle du lien interpersonnel, que le terme de tendresse, inconnu en ce sens au $\mathrm{XVI}^{\mathrm{e}}$ siècle, viendra désigner au tournant du siècle suivant ${ }^{13}$. On sait aussi ce que le modèle du Tendre doit, par l'intermédiaire de L'Astrée d'Honoré d'Urfé, à l'héritage

10. Composé au sein du cercle de Madeleine de Scudéry, entre l'été 1653 et l'hiver 1654, le manuscrit rassemble un choix de billets galants et de poésies échangés entre les principaux animateurs du groupe, l'académicien Valentin Conrart (sous le nom de Théodamas), son ami, coreligionnaire et futur confrère Paul Pellisson (Acante), enfin Madeleine de Scudéry elle-même (Sapho) - auxquels s'adjoignent ponctuellement d'autres épistoliers. Nous en avons procuré une édition critique: Madeleine de Scudéry, Paul Pellisson et leurs amis, Les chroniques du Samedi. Suivies de pièces diverses (1653-1654), Alain Niderst, Delphine Denis et Myriam Maître (éd.), Paris, Éditions Honoré Champion, coll. "Sources classiques», 2002. Désormais les références à cet ouvrage seront indiquées par le sigle «CS», et placées entre parenthèses dans le corps du texte.

11. Clélie, que le sous-titre d'Histoire romaine apparente au genre du roman héroïque, est publiée à Paris, chez Augustin Courbé: la livraison de ses dix volumes s'échelonne de 1654 à 1660. Nous citerons dans l'édition procurée par Chantal Morlet-Chantalat, Clélie, Paris, Éditions Honoré Champion, coll. «Sources classiques», 2001-2004 pour les quatre premières parties (la cinquième à paraître en 2005). Désormais les références à cet ouvrage seront indiquées par le sigle « $\mathrm{C}$ », et placées entre parenthèses dans le corps du texte.

12. Notamment, bien sûr, Philippe Ariès et Georges Duby (dir.), Histoire de la vie privée, t. III, sous la direction de Roger Chartier, De la Renaissance aux Lumières, «Formes de la privatisation », Paris, Éditions du Seuil, coll. «L'univers historique», 1986, p. 159394. Et aussi Maurice Daumas, La tendresse amoureuse, XVI ${ }^{e}$-XVIII ${ }^{e}$ siècles, Paris, Éditions Perrin, 1996.

13. Le Dictionnaire universel de Furetière retient cette acception: "Sensibilité du cœur et de l'âme. La délicatesse du siècle a renfermé ce mot dans l'amour et dans l'amitié. Les amants ne parlent que de tendresse de cœur, soit en prose, soit en vers; et même ce mot signifie le plus souvent amour; et quand on dit, J'ai de la tendresse pour vous, c'est-à-dire, j'ai beaucoup d'amour.» (Antoine Furetière, entrée «Tendresse», Dictionnaire universel, 1690) 


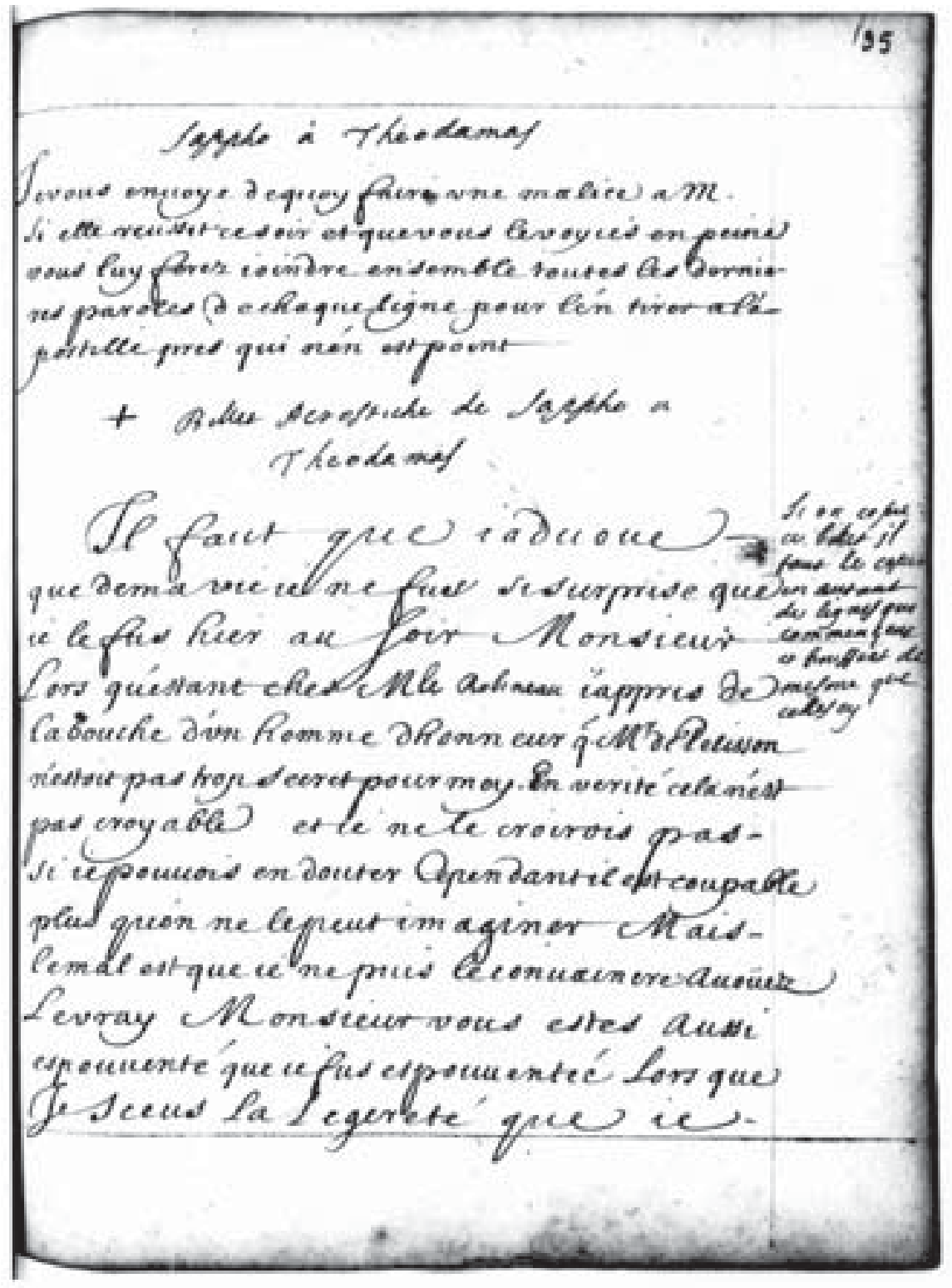

Fig. 2. Les chroniques du Samedi (1653-1654), Manuscrit 15156, Bibliothèque de l'Arsenal de Paris, fo 95 , coll. Bibliothèque nationale de France. 
courtois, moyennant bien des réaménagements ${ }^{14}$. À compter des années 1640 enfin, les ruelles féminines allaient offrir une scène nouvelle à la sociabilité mondaine: des formes de relations inédites s'actualisèrent alors, qui, au sein d'une civilité partagée dans un cercle choisi, permettaient peu à peu de nouer des amitiés électives centrées autour de ces femmes de prix - qu'on appela Précieuses.

Si l'on n'inaugure donc pas de toutes pièces une manière de ressentir, il n'en reste pas moins que tenter d'en fixer le discours, et d'en mettre en scène l'acte de naissance, peut s'interpréter comme un geste fondateur qu'il convient d'abord de restituer à ce premier mouvement. Or, deux «récits des origines » enserrent l'apparition de la célèbre Carte de Tendre (fig. 3), ainsi présentée comme un événement dont la dynamique n'importe pas moins que le contenu propositionnel. Le premier en date des deux textes, on l'a dit, est le manuscrit des Chroniques du Samedi, trop souvent interrogé comme simple document où se lirait en toute transparence l'histoire anecdotique de cette invention. Pourtant ce «témoignage» qui nous ferait entrer dans la fabrique de l'œuvre ne nous est transmis que sous une forme déjà médiatisée, mêlant avec une duplicité consciente les notes marginales, neutres et informées, du «Chroniqueur» directement concerné, et le corps du recueil proprement dit, constitué des billets galants échangés entre des protagonistes qui ne s'écrivent que sous couvert de pseudonymes - il est vrai parfaitement déchiffrables par tous les intéressés. S'il est licite, et d'ailleurs en quelque sorte prévu par le texte, de verser cette séquence au compte des archives de la création romanesque, encore fautil prendre toute la mesure de ce travail de figuration littéraire effectué dans le manuscrit, qui interdit de replier sans précaution «l'œuvre sur le référent ${ }^{15}$ »: de l'un à l'autre reste du jeu. De quoi s'agit-il en effet? D'une amitié naissante (entre Madeleine de Scudéry et Paul Pellisson) dont les «progrès » vers plus d'intimité, partant plus de sincérité, non seulement se publient dans le recueil publication contrôlée et réservée, mais effective malgré tout, puisque celui-ci circule dans le groupe, parfois même au-delà - mais encore recourent pour s'exprimer à d'ingénieuses galanteries, billets enjoués, métamorphoses para-

14. Voir Gerhard Penzkofer, L'«art du mensonge». Erzählen als barocke Lügenkunst in den Romanen von Mademoiselle de Scudéry, Tübingen, Gunter Narr, 1998, p. 162-211.

15. Selon la mise en garde de Bernard Beugnot, «Edipe et le Sphinx. Des clés", dans La mémoire du texte. Essais de poétique classique, Paris, Éditions Honoré Champion, coll. «Lumière classique », 1994, p. 227-242. 


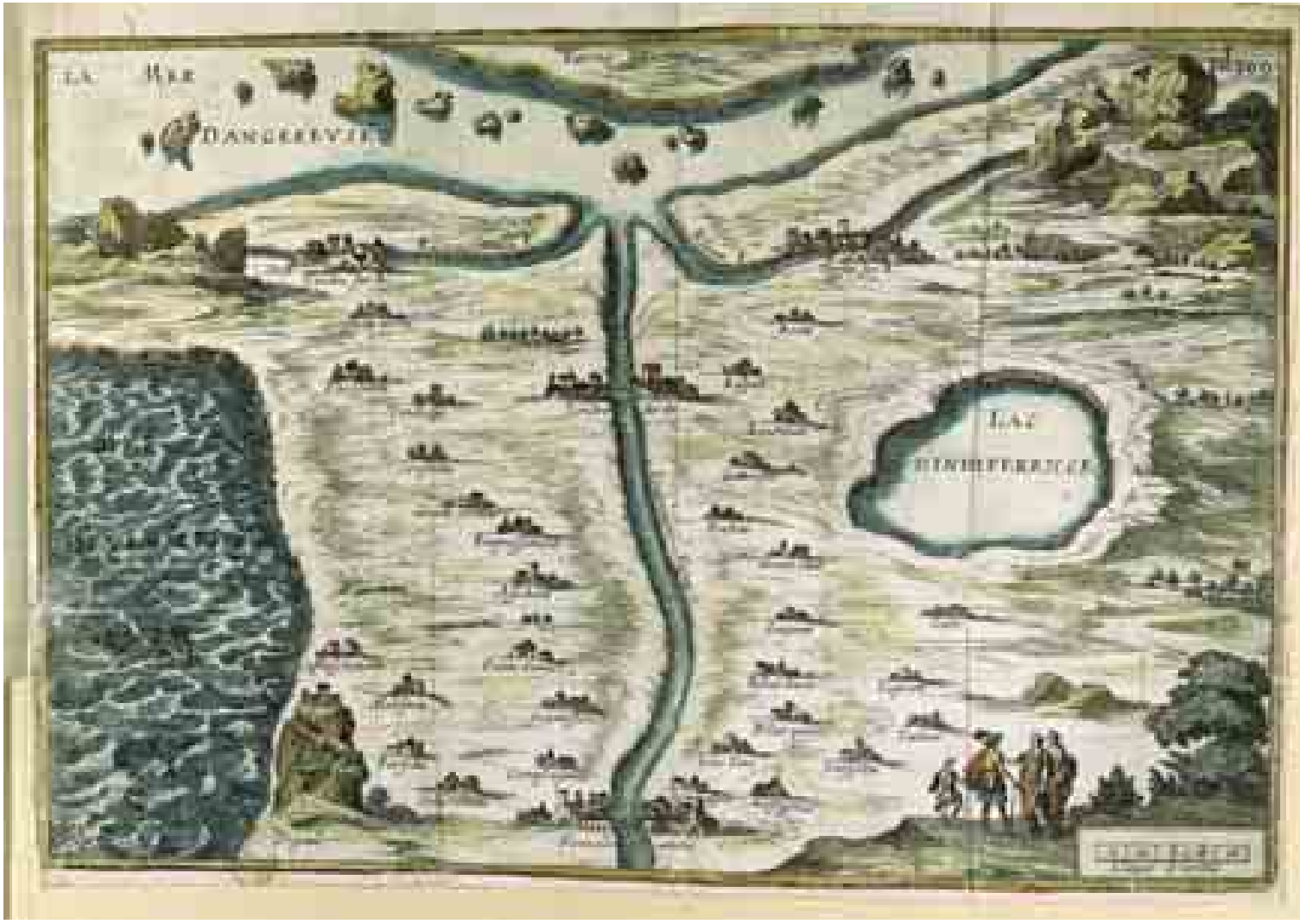

Fig. 3. François Chauveau, La carte de Tendre, dans Madeleine de Scudéry, Clélie, première partie, 1654 , coll. Bibliothèque nationale de France.

doxales, allusions et jeux de mots. L'échange occupe deux longs billets rédigés dans le prolongement d'une conversation au Samedi de Sapho. Un «Argument» scrupuleusement consigné en note, écrit de la main de Pellisson (Acante) en fournit une précise contextualisation, et prévoit même, à terme, de «conserver soigneusement» les deux dessins successifs de la Carte:

Argument de ce qui suit.

En une conversation du Samedi, Sapho ayant fait sur le sujet de l'amitié une distinction entre ses nouveaux amis, ses particuliers amis et ses tendres amis, Acante demanda de quel rang il était, et on lui dit que c'était des particuliers. Il s'avisa de demander s'il y avait bien loin de Particulier à Tendre, et si un homme qui marcherait toujours en diligence pourrait espérer d'y arriver depuis le mois de novem- 
bre où on était jusques au mois de février qui était celui où finissaient les six mois que Sapho avait pris pour l'éprouver. Il lui fut répondu que ce serait suivant la route qu'il tiendrait, parce que s'il manquait le chemin il n'y arriverait jamais. Il demanda combien il y avait de routes. On lui dit qu'on y pouvait aller par eau, par terre et par air, et qu'il choisissait laquelle des trois il voulait: il dit que c'était la dernière comme la plus courte et qu'il trouverait plus tôt l'invention de voler. Sur quoi il fut parlé de plusieurs personnes qui avaient cru que cela n'était pas impossible. Sur quoi il écrivit deux jours après le billet suivant.

Cette galanterie au reste étant poussée plus avant donna naissance à la fameuse carte de Tendre, qui depuis a été mise dans le premier volume de Clélie.

Il faudra attacher ici ou après ce billet le premier projet de la Carte de Tendre qui fut fait ensuite au premier Samedi par Sapho en se jouant et y mettre aussi la carte qui a été gravée et imprimée depuis afin de conserver soigneusement cet original et de faire voir ce qu'on y a changé depuis, qui est fort peu de chose. (CS, p. 126)

Dans le premier billet, enchaînant plaisamment avec cette conversation, Pellisson s'imagine en mouche volante pour hâter le voyage de Particulier à Tendre; il demande alors de plus amples précisions:

[...] je ne souhaite point d'être un aigle, non pas même un roitelet, et je me contenterais d'être une mouche: aussi bien ai-je déjà quelque rapport avec cet animal. Je ne fais guère de bruit. J'importune fort souvent mais je ne blesse jamais. J'aime furieusement les douceurs et bien qu'on m'accuse quelquefois d'être une fine mouche, il n'y a point d'autre bête qui se laisse plus aisément attraper que moi. Enfin nonobstant les autres incommodités qui s'y pourraient rencontrer, je vous l'avoue, je m'accommoderais extrêmement de cette métempsycose, pourvu que tout doucement et sans qu'on prît garde à moi j'arrivasse bientôt du pays de Particulier à celui de Tendre, où j'aspire depuis si longtemps. Mais, Mademoiselle, quand je serai ainsi métamorphosé et métempsychosé, de quoi je ne désespère pas, le moyen de ne me point égarer en chemin? Voulez-vous qu'une pauvre mouche aille faire tout le tour du monde sans savoir où elle va? Il ne faut qu'un coup de main pour la perdre. Le premier prince fainéant qu'elle trouvera (et vous savez qu'on en trouve assez) se divertira à la faire mourir, et au pis aller le froid de l'hiver ne manquera pas à la tuer si elle n'a fait son voyage avant les grandes gelées. Enseignez-lui donc de grâce, Mademoiselle, quelle route elle doit tenir, par quels lieux elle doit passer, quels dangers elle doit craindre et dites-lui le secret pour achever promptement sa course. (CS, p. 127-128)

La réponse de Madeleine de Scudéry poursuit sur le même ton, différant sans s'y refuser l'envoi «d'une petite carte de ce pays-là » (CS, p. 130). Du délai de six mois à l'épreuve duquel l'entrée de Pellisson dans Tendre est suspendue, les Chroniques marqueront ainsi les étapes, scandées par les divertissements 
littéraires du groupe. Le jeu s'étend cependant dans le petit cercle. D'autres candidats au voyage se déclarent, occasion pour rivaliser à leur tour d'ingéniosité dans l'accomplissement imaginaire du parcours: Billets-doux et Jolis-Vers seront autant de manifestations séduisantes de Grand-Esprit, tandis que les plus anciens admirateurs de Madeleine de Scudéry font de Constante-Amitié un droit d'accès prioritaire à Tendre. Sapho répond à chacun en public, assignant les places et rabattant les revendications abusives de tel ou tel. À Acante seul, elle signalera par des formules détournées l'arrivée imminente de l' " opiniâtre ${ }^{16}$ » voyageur au Royaume de Tendre ${ }^{17}$. On le voit, de cette intimité à venir, le modèle qui se met en place ne cherche ni le secret, ni l'expression directe. De même que le chemin s'avère plus long qu'il ne semblait, mais d'autant plus agréable à parcourirr ${ }^{18}$, de même le tour figuré ménage, dans le délai nécessaire à l'application personnelle, le plaisir accru de deviner, celui redoublé d'offrir «une explication bien déliée et bien subtile» du demi-mot - parodie galante de la tradition humaniste du commentaire érudit:

J'ai $[\ldots]$ fait de très grands et de très longs commentaires sur ce que vous me faites la grâce de me dire, que je suis plus éloigné que jamais de Nouvelle Amitié. Il me semblait qu'il eût été mieux de dire plus proche de Tendre et je vous ai accusée deux ou trois cents fois d'avoir eu la dureté d'éviter ce mot. Toutefois enfin, comme un critique bien expert quand il ne peut pas trouver autrement son compte dans son auteur, j’ai inventé une explication bien déliée et bien subtile de laquelle je voudrais bien que vous demeurassiez d'accord. Je dis donc qu'il est beaucoup mieux que je sois bien loin de Nouvelle Amitié que non pas si j'étais bien proche de Tendre (excusez l'expression, c'est du français de commentaire), et ma raison est que si un homme était assez heureux pour être dans Tendre même, il pourrait dire qu'il est

16. Telle est l'épithète de Pellisson dans les Chroniques du Samedi.

17. «[...] je puis vous assurer sans mensonge, que jamais nul ami, amant, n’a été si près de Tendre que vous: après cela, n’en demandez pas davantage si vous ne voulez être refusé. Au reste j'ai encore à vous avertir, que je n'ai jamais eu la peine de dire à ce petit nombre d'amis privilégiés que j'ai eu en ma vie, qu'ils étaient arrivés à Tendre: car à vous dire la vérité, quand on y est on s'en aperçoit, et ceux qui ne s'en aperçoivent pas, ne sauraient jamais qu'ils y auraient été. » (CS, p. 240) et «Mettez-vous donc l'esprit en repos s'il est vrai que vous l'ayez en peine, et croyez que vous ne fûtes jamais si loin de Nouvelle Amitié que vous êtes.» (CS, p. 255)

18. «Au reste j’ai connu quelques gens en ma vie qui avaient été de Particulier à Tendre et qui y avaient même été par le chemin le plus long. Cependant ils m’assurèrent qu'ils ne s'étaient pas ennuyés de sa longueur et qu'il y avait mille agréables choses à voir en y allant, qui divertissaient assez.» (CS, p. 130) 
plus loin que jamais de Nouvelle Amitié. Mais il ne pourrait pas dire sans incongruité qu'il est plus proche de Tendre que jamais, car on n'est point proche des lieux dans lesquels on est. En cas que vous jugiez cette interprétation raisonnable, je veux bien mourir si je n’aime mieux l'avoir trouvée que toutes celles des Lipse, des Heinsius et des Saumaise. («Acante à Sapho», CS, 256-257)

De ce premier récit d'invention, quelques lignes de force se dégagent. C'est dans le cadre d'une compagnie choisie, où le travail de «distinction" (CS, p. 126) se fonde sur les degrés de proximité affective, qu’a pu être proposée la formule de l'amitié tendre. Amitié, et non amour, comme les proches de Sapho l'ont parfaitement compris ${ }^{19}$, de surcroît exclusive de tout autre mode d'attachement, et susceptible de réelles jalousies. D’une sociabilité plus généralement consentie, Madeleine de Scudéry isole donc un lien d'élection, auquel l'accès supposé désirable n'est en rien garanti. S'il appartient au secret des cœurs d'en éprouver la réussite, le modèle est cependant publiable, convertissant alors la description galante en code de comportement moral. Prescriptif? Sans doute moins qu'il n'y paraît, car on ne s'engage que de plein gré pour pareilles tribulations. Normatif? À coup sûr non, puisqu'il n'entend pas s'imposer comme l'unique modalité des relations interpersonnelles, et demeure au contraire réservé au petit nombre de ces voyageurs exigeants: beaucoup d'ailleurs s'égarent en route, dont la Gazette de Tendre ${ }^{20}$ évoquera avec humour les regrettables errances. Enfin, ces jeux d'esprit dont la productivité encombrante, voire lassante, semble contredire la légèreté affichée, ont pu paraître à ce point mériter une «histoire » qu'il leur fallut un «Chroniqueur» dévoué: le recueil manuscrit s'y emploie, rassemblant pour d'aléatoires lecteurs une archive à son tour en attente d'historiens, de glossateurs prolixes et de critiques soupçonneux. Savoir rétrospectivement que les Chroniques inauguraient enfin, sur le plan strictement biographique, une amitié indéfectible de plus de quarante ans entre les deux principaux auteurs du recueil ne va pas sans troubler quelque peu l'injonction d'une séparation méthodique entre la création littéraire et la vie vécue dont il

19. Par exemple, dans ce madrigal de Jacques de Ranchin, également conservé dans les Chroniques: "Car avant que la Carte fût,/Le moyen alors de comprendre/Qu'il était une amitié tendre,/Qui diffère autant de l'amour/Que la nuit diffère du jour? »(CS, p. 261)

20. Conservée dans le Recueil Conrart (Bibliothèque de l'Arsenal, Ms. 5414, fos 147158). Nous l'avons reproduite dans les annexes de l'édition citée des Chroniques du Samedi, p. 306-321. 
serait «scientifiquement» plus confortable de préserver l'étanchéité - mais que cette littérature elle-même nous invite au contraire à mettre en question de front.

Le second récit d'invention, bien connu, accompagne dans le premier volume de Clélie l'apparition de la Carte de Tendre gravée par François Chauveau, immédiatement suivie de sa glose - dans la plus riche tradition herméneutique de l'ecphrasis ${ }^{21}$. Cette fois encore, Madeleine de Scudéry avait pris soin d'en circonstancier l'événement. Une longue conversation, écho stylisé de celle tenue au Samedi de Sapho, avait en effet introduit, une centaine de pages plus haut, le thème de la tendresse. Il s'agissait de définir sa véritable nature au moyen de «peintures » fidèles, pour résister aux usages inflationnistes du mot, sources d'une grave dévaluation sémantique ${ }^{22}$. Abus terminologique qui vient signaler la désaffection des conduites, rapportées à une civilité indistincte aussi nécessaire qu'aisément falsifiable. Pour redonner force au mot, que sa récente introduction dans le lexique exposait aux redoutables effets de mode, il faut donc en passer par le travail inverse de séparation - de distinction: mieux que par l'analyse sémantique $a b$ abstracto, c'est dans la fine description des comportements que cet essai de définition ${ }^{23}$ entend trouver sa pertinence. Ainsi mots et choses verraient-ils comblé l'écart qui les disjoint dans le toutvenant des relations humaines. Deux exposés se succéderont, confiés aux plus experts en la matière, et distribués selon les règles de la bienséance (convenance des caractères, adhésion aux valeurs supposées communes) : à Clélie le soin de peindre l'amitié tendre, tandis qu'Aronce se fera le chantre inspiré de la tendresse amoureuse. Pour l'un comme pour l'autre, il semble bien que le «tendre» fasse mieux que caractériser une modalité particulière du lien amical

21. Voir Anne-Élisabeth Spica, Savoir peindre en littérature. La description dans le roman au XVII siècle: Georges et Madeleine de Scudéry, Paris, Éditions Honoré Champion, coll. «Lumière classique », 2002, p. 332-340.

22. « - Tant de gens s'en servent aujourd'hui, répliquai-je [Célère, narrateur de ce récit pour la princesse des Léontins], qu'on ne saura bientôt plus sa véritable signification. - Je voudrais pourtant bien empêcher, dit Clélie, que ce mot qui signifie une chose si douce, si rare, et si agréable, ne fût profané; cependant, comme l'a dit Célère, tout le monde s'en sert aujourd'hui. - En mon particulier, répliqua Sozonisbe, je vous promets de ne m'en servir jamais si je ne le dois, pourvu que vous veuilliez bien me faire entendre sa véritable signification. » (C, p. 115)

23. Au double sens du terme, littéral (établissement de frontières) et logique (définition dite «imparfaite» par la description non exhaustive des propriétés en jeu). 
ou amoureux : il en achève les virtualités sémantiques, le syntagme venant alors désigner la forme accomplie, seule digne de ce nom, de l'amitié ou de l'amour. C'est qu'elle met en jeu la dimension sensible de la personne ${ }^{24}$, gage de la densité et de la richesse de la relation à l'autre:

Mais pour bien définir la tendresse, je pense pouvoir dire que c'est une certaine sensibilité de cœur, qui ne se trouve presque jamais souverainement, qu'en des personnes qui ont l'âme noble, les inclinations vertueuses, et l'esprit bien tourné, et qui fait que, lorsqu'elles ont de l'amitié, elles l'ont sincère, et ardente, et qu'elles sentent si vivement toutes les douleurs, et toutes les joies de ceux qu'elles aiment, qu'elles ne sentent pas tant les leurs propres. C'est cette tendresse qui les oblige d'aimer mieux être avec leurs amis malheureux, que d'être en un lieu de divertissement; c'est elle qui fait qu'ils excusent leurs fautes, et leurs défauts, et qu'ils louent avec exagération leurs moindres vertus. C'est elle qui fait rendre les grands services avec joie, qui fait qu'on ne néglige pas les petits soins, qui rend les conversations particulières plus douces que les générales, qui entretient la confiance, qui fait qu'on s'apaise aisément, quand il arrive quelque petit désordre entre deux amis, qui unit toutes leurs volontés, qui fait que la complaisance est une qualité aussi agréable à ceux qui l'ont, qu'à ceux pour qui on l'a, et qui fait enfin toute la douceur, et toute la perfection de l'amitié. (c, p. 118)

Égards, attentions, complaisance, délicatesse en sont autant de manifestations qu'on voudrait croire impossibles à singer, et qu'en retour une «âme tendre» saura reconnaître sans risque de s'y méprendre:

On peut sans doute se déguiser quelquefois; mais ce ne peut être pour longtemps; et ceux qui se connaissent en tendresse, ne s'y sauraient jamais tromper. En effet toutes les paroles, tous les regards, tous les soins, et toutes les actions d'un amant qui n'a point le cœur tendre, sont entièrement différentes de celles d'un amant qui a de la tendresse; car il a quelquefois du respect sans avoir d'une espèce de soumission douce, qui plaît beaucoup davantage, de la civilité sans agrément, de l'obéissance sans douceur, et de l'amour même, sans une certaine sensibilité délicate, qui seule fait tous les supplices, et toutes les félicités de ceux qui aiment, et qui est enfin la plus véritable marque d'une amour parfaite. (C, p. 120-121)

Dès lors, galanterie et tendresse se confortent sans se confondre: "La tendresse a encore cela de particulier qu'elle lui donne même je ne sais quel caractère de galanterie qui la rend plus divertissante; elle inspire la civilité et

24. La famille lexicale de l'adjectif sensible, et son champ sémantique, sont omniprésents dans cette conversation. 
l'exactitude à ceux qui en sont capables. » (C, p. 117) Au-delà des signes partagés de la civilité, c'est dans le secret des coeurs que s'en éprouve la valeur.

Présentés en diptyque, ces deux volets de la définition du Tendre dessinent un espace commun où l'amour et l'amitié se rencontrent, distingués cependant nettement l'un de l'autre ${ }^{25}$. Une même sensibilité se voit dès lors créditée aux hommes et aux femmes, ouvrant à toute la gamme des relations intimes. On comprend mieux ainsi les diverses lectures auxquelles a pu se prêter la célèbre Carte, dont Clélie fournit, après les Chroniques, le récit d'origine. L'«argument» initial, déplacé dans ce nouveau contexte, nous reconduit à la distinction des formes d'amitié désormais opérée par l'héroïne du roman : c'est pour répondre à la requête d'Herminius - nouvel avatar d'Acante-Pellisson - reprise en chœur par le groupe, que Clélie relève la gageure de «dresser la carte d'un pays, dont personne n'avait encore fait de plan. » (c, Ire partie, livre I, p. 180) Double défi que cette commande, dont la livraison aura été précautionneusement consignée en amont, à toutes les étapes de son invention; c'est que, s'en inquiète Clélie, les périls encourus par une divulgation décontextualisée de cette «bagatelle» ne sont pas négligeables:

Je sais bien, poursuivit-elle, que ceux qui savent que cela a commencé par une conversation qui m'a donné lieu d'imaginer cette carte en un instant, ne trouveront pas cette galanterie chimérique ni extravagante; mais comme il y a de fort étranges gens par le monde, j'appréhende extrêmement qu'il n'y en ait qui s'imaginent que j'ai pensé à cela fort sérieusement, que j'ai rêvé plusieurs jours pour le chercher, et que je crois avoir fait une chose admirable. Cependant c'est une folie d'un moment, que je ne regarde tout au plus que comme une bagatelle qui a peut-être quelque galanterie, et quelque nouveauté, pour ceux qui ont l'esprit assez bien tourné pour l'entendre. (c, p. 185-186)

25. Plus loin dans le roman, une autre conversation d'égale importance reprendra sur nouveaux frais cette question : le glissement progressif de l'amitié à l'amour, évoqué par Herminius, prétendra fonder la perfection du sentiment amoureux sur la base de celle de l'amitié. «[...] l'amour et l'amitié se mêlent comme deux fleuves, dont le plus célèbre fait perdre le nom à l'autre. Mais après tout, les eaux du plus petit y sont effectivement aussi bien que celles du plus grand; de sorte qu'encore qu'un ami qui est devenu amant, dise toujours alors qu'il a de l'amour, et ne dise plus qu'il a de l'amitié, il est pourtant certain que ces deux sentiments-là sont dans son cœur, quoiqu'il ne les puisse presque plus discerner; et il est constamment vrai qu'une amour de cette espèce, est plus parfaite que l'autre. » (C, III ${ }^{\mathrm{e}}$ partie, livre V, p. 129) 
Que cette «morale d'amitié», ainsi que la désigne Célère, ait pu être comprise comme un itinéraire amoureux - et souvent contesté comme tel, qu'on le jugeât hypocritement licencieux ${ }^{26}$ ou prude à l'excès ${ }^{27}-$ ne relève pas, loin s'en faut, du contresens. Les efforts déployés par Madeleine de Scudéry pour en régler l'interprétation sont bien le symptôme d'une difficile stabilisation : l'invention de l'amitié tendre et sa précise cartographie couraient inévitablement le risque d'être appliquées à l'autre espace de l'intimité affective. Les lecteurs en effet ne pouvaient manquer de se souvenir qu'Aronce avait fait l'éloge de la tendresse amoureuse; d'autre part, plusieurs romans du début du siècle entretenaient l'ambiguïté, renvoyant dans leurs titres les amours des héros à la catégorie alors englobante de «l'honneste amitié», modèle éprouvé sur lequel penser le parfait amour ${ }^{28}$. Surtout, la définition même du roman imposait pareil déplacement: la formule proposée quelques années plus tard par Pierre-Daniel Huet («des histoires feintes d'aventures amoureuses, écrites en prose avec art, pour le plaisir et l'instruction des lecteurs ${ }^{29} »$ ) fait de la matière amoureuse une composante constitutive du genre. L'histoire principale d'Aronce et de Clélie elle-même, qui entendait pourtant se tenir en deçà « des dernières bornes de l'amitié » (C, Ire partie, p. 184), confirmera bien vite la validité de cette définition. Comment alors ne pas être tenté de lire la Carte comme un vaste programme narratif, dont les diverses histoires enchâssées du roman actualiseront les possibles? Mieux encore qu'avec le couple de ces parfaits amants, le lecteur trouvera dans la $\mathrm{III}^{\mathrm{e}}$ partie du roman, avec l'«Histoire d'Herminius et de Valérie» une illustration exemplaire de la cohérente plasticité de Tendre.

26. On peut relire notamment Nicolas Boileau, Satire X, v. 158-164, Jean-Pierre Collinet (éd.), Paris, Éditions Gallimard, coll. «Poésie», 1985 [1657], p. 127. Madeleine de Scudéry fut même par d'autres chansonnée en «maquerelle»...

27. Des chemins plus directs vers Tendre, moins éthérés, avaient été proposés sous forme ironique: la voie érotique des «Bijoux» est ainsi pour Segrais la plus sûre. Voir à ce sujet Jean Regnault de Segrais, Diverses poésies, Paris, Antoine de Sommaville, 1658.

28. L'exemple de L'Astrée, roman fondateur pour la création scudérienne, s’impose ici avec force; on rappellera le titre sous lequel parut en 1607 (chez Toussaint Du Bray) la première partie de l'ouvrage: Les douze livres d'Astrée, où, par plusieurs Histoires et sous personnes de Bergers et d'autres, sont déduits les divers effets de l'honnête amitié. Sur la prégnance du paradigme de l'amitié dans cette période, voir Maurice Daumas, La tendresse amoureuse, p. 94-118.

29. Lettre de Monsieur Huet à Monsieur de Segrais. De l'origine des romans (Préface de Zaïde), Fabienne Gégou (éd.), Paris, Éditions Nizet, 1971 [Paris, Claude Barbin, 1670], p. 46-47. Le Tasse avait déjà indiqué ce lien du romanzo à la topique amoureuse. 
Se croyant d'abord ami de Valérie, Herminius fera peu à peu l'expérience du sentiment amoureux. C'est au terme de tendresse qu'il appartiendra de ménager la transition:

[...] Herminius ayant pris le mot d'amitié en haine, et n'osant proposer celui d'amour, il ne se servait que de celui de tendresse pour exprimer les sentiments qu'il avait pour elle. (C, III ${ }^{\mathrm{e}}$ partie, livre I, p. 96)

avant que soit enfin désignée, au risque de la déclaration d'amour, la nature de cette affection:

Querellez-moi donc Madame, reprit-il, car je vous assure que j’ai voulu dire le mot d'amour, et qu'il n'y en a point d'autre en notre langue, qui puisse exprimer ce que je sens pour vous [...]. Je ne sais même, poursuivit-il, si vous ne le savez point devant moi; car je vous avoue que quelque tendresse que j'aie pour vous, la pureté de mon affection, faisait que je la prenais pour de l'amitié; mais à n'en mentir pas, son ardeur me la fait aujourd'hui bien connaître. (C, p. 96-97)

La vertueuse Valérie aura beau essayer de s'en tenir à la formule moins coûteuse de l'amitié tendre ${ }^{30}$, Herminius ne veut plus de ce flou sémantique:

Ha Madame [...], ne me défendez point la plus douce et la plus agréable parole du monde, pour ceux qui ont dans le cœur la passion qu'elle exprime. Car enfin le mot d'amour a quelque charme secret qui émeut le cœur d'un amant qui le prononce, et qui touche celui de la personne qui l'entend, quand elle a une véritable tendresse dans l'âme. Celui d'affection est un mot douteux, qui convient à l'amitié comme à l'amour; celui de tendresse tout obligeant qu'il est, peut être aussi employé à ces deux choses. (C, p. 98)

Les étapes de la Carte une à une franchies ${ }^{31}$ "Valérie s'adoucissant peu à peu, permit à Herminius de l'aimer » (C, p. 99), à la condition de ne jamais en prononcer le mot... Comme il faut pourtant faire avancer l'intrigue, et malgré

30. "Je vous promets [...] de faire pour vous tout ce que je pourrai, et d'attribuer toujours à la tendresse de votre amitié, tous les soins que vous aurez pour moi, tant que vos paroles ne s'opposeront pas à l'opinion avantageuse que je veux avoir de vous.» (c, p. 98)

31. «Mais à la fin Herminius fit tant de choses obligeantes, que le cœur de Valérie en fut attendri, car il prenait tous ses désirs, quand il les pouvait deviner; il contribuait de cent manières différentes à son divertissement; il rendait office à toutes les personnes qu'elle aimait; il n'avait plaisir en aucun lieu que lorsqu'il la voyait; il était le plus respectueux de tous les hommes; il lui écrivait mille agréables douceurs, dont elle ne se pouvait offenser [...].» (c, p. 99) 
tout (se) parler d'amour, l'expédient imaginé par le couple ne surprend pas, qui choisit de cacher sous «une civilité universelle [...] l'estime particulière» (C, p. 100) qui les unit: derrière la galanterie des comportements se décèle l'intimité du Tendre. C'est aux ressources de l'écriture stéganographique que sera confié le secret des cœurs, publiquement avouable sous les espèces de l'amitié, mais réservé en sa véritable essence:

Herminius [...] convint avec Valérie, que le mot d'amitié voudrait dire amour entre eux, soit qu'il lui parlât, ou qu'il lui écrivît. Et en effet la chose étant ainsi concertée, Herminius écrivait des billets à Valérie, qui ne passaient que pour des billets d'amitié, et qui étaient pourtant des billets d'amour. (C, p. 99)

Ambivalence pourtant périlleuse du stratagème: pareil mode d'expression ne risque-t-il pas en effet de ruiner la distinction récemment établie entre ces deux modalités de la relation interpersonnelle? Aussi faut-il l'assortir d'un chiffre qui métamorphosera aux yeux des intrus cette correspondance amoureuse en «galimatias d'amitié » (C, p. 133):

[...] lorsqu'ils étaient tombés d'accord entre eux, que dans leurs billets le mot d'amitié voudrait dire amour, [...] Valérie avait demandé à Herminius comment elle ferait, lorsque dans un même billet elle voudrait se servir du mot d'amitié, pour signifier simplement amitié. De sorte que comme Herminius est fertile en inventions, il lui avait dit que lorsque le mot d'amitié ne voudrait point dire amour, il faudrait l'écrire avec une grande lettre au commencement, et que lorsqu'il voudrait dire amour, il faudrait l'écrire avec une petite seulement. (C, p. 134-135)

L'artifice graphique sera vite découvert, mais l'irréprochable conduite des protagonistes parviendra à le reconvertir, pour les indiscrets, en «malices innocentes» et "simple jeu d'esprit» (c, p. 138 et 139). Ainsi l'aventure amoureuse déguisée en ingénieuse galanterie peut-elle déjouer la curiosité publique, et de nouveau réserver au for privé des seuls intéressés la juste qualification du sentiment: bagatelle dont la duplicité protège en réalité l'authenticité vécue... Comment ne pas rétrospectivement reporter sur la Carte de Tendre la possibilité de pareille réserve? La boucle se referme, de surcroît, en son point d'origine, puisque l' «Histoire d'Herminius et de Valérie» emprunte elle aussi quelquesuns de ses épisodes aux Chroniques du Samedi. Dans le recueil manuscrit, en effet, Acante-Pellisson avait galamment pu s'acquitter de deux injonctions contradictoires ${ }^{32}$ par le truchement d'un billet acrostiche à Sapho, avouant sa ruse

32. Faire tenir sans tarder à Madeleine de Scudéry des vers de son ami Thrasile (le poète Samuel Isarn), auquel il avait promis de laisser la priorité de l'envoi : deux « exactitudes » simultanément intenables. 
à la lettre sans pour autant trahir la promesse faite à l'ami trop zélé; réponse de la bergère au berger, Sapho l'avait ensuite «attrapé» par une astuce graphique de plus grande finesse encore (CS, p. 94-95). Ces deux billets, accompagnés du commentaire de leurs effets (déception et brouille passagères, plaisir et soulagement lors de la résolution de l'énigme, connivence accrue entre les épistoliers), se retrouvent à peine modifiés dans la troisième partie du roman (C, p. 104-105 et 112-113) 33. Autant d' «agréables mensonges » qui délivrent, à qui sait déchiffrer, la vérité secrète qu'une formulation directe aurait paradoxalement interdite. Épigramme en vers cachée sous la prose, aveux tout à la fois lisibles et cryptés, dont l'acrostiche livrera le fin mot, on conviendra avec Herminius que «les apparences sont bien souvent fort trompeuses»... (C, p. 105) À aucun moment cependant, la galanterie n'aura fait sécession avec la tendresse. Car si c'est du côté de Grand-Esprit ou d'Empressement, d'Exactitude ou d'Obéissance qu'il faut en l'occurrence situer cette série d'échanges, on sait par ailleurs nos héros embarqués pour un voyage amoureux.

On se gardera d'en faire trop vite l'application aux épistoliers des Chroniques, puisque c'est sous le masque de noms romanesques qu'ils avaient souhaité figurer: médiation littéraire explicite (Sapho, Théodamas, Agélaste, Thrasile et tant d'autres sont tout droit sortis de la précédente fiction scudérienne, Artamène ou Le Grand Cyrus; quant à Acante, sa fortune poétique est assurée depuis plusieurs décennies, et La Fontaine en prolongera peu après la postérité ${ }^{34}$ ) qui sert de garde-fou contre semblable tentation.

Le Tendre confirme donc, à plus d'un titre, l'analyse de Niklas Luhmann qui voit dans les mutations du code amoureux la «constitution d'un médium de communication généralisé au plan symbolique, auquel est assignée la tâche spécifique de permettre, de cultiver et de favoriser le traitement communicationnel de l'individualité». En partie accompli au milieu du XVII ${ }^{\mathrm{e}}$ siècle, ce processus aurait contribué à mettre en place la pensée d'un «monde privé commun $^{35} »$, en distinguant des anciennes solidarités ainsi que de l'indivis des

33. Palimpseste supplémentaire, plus loin dans l'histoire, Valérie croyant Herminius mort et inconstant, demandera copie de tous les billets échangés entre Clélie et Herminius qu'elle soupçonne d'être amants, ainsi que de la Carte de Tendre. La lecture de cet ensemble, et l'examen attentif des chemins oublieux de la Carte - jusqu'au Lac d'Indifférence - la conforteront dans sa douloureuse méprise. (C, p. 165-169)

34. Voir sur ce point Judd D. Hubert, «La Fontaine et Pellisson, ou le mystère des deux Acante", Revue d'histoire littéraire de France, vol. 66, n 2, avril-juin 1966, p. 223-237.

35. Niklas Luhmann, Amour comme passion, p. 26 et 28. 
rapports sociaux, de plus en plus diversifiés, une relation progressivement plus personnelle, confidentielle. Mais, à côté du modèle de «l'amour passion » que privilégie son étude, et qui trouvera son accomplissement dans la posture romantique du $\mathrm{XIX}^{\mathrm{e}}$ siècle — jusqu'en ses avatars modernes - la voie «moyenne» de Tendre a pu constituer une alternative optimiste aux conflits sans cesse réaffirmés de l'Amour et de la Raison, et offrir un espace de médiation où l'amour et l'amitié, désormais nettement différenciés, partageraient néanmoins la même exigence d'un lieu intime, authentique, librement consenti, discrètement ménagé au cœur des pratiques de sociabilité. Et si la galanterie bienséante continue de prendre en charge les jeux de rôles inégalitaires entre hommes et femmes, de souffrir exagérations et paradoxes ${ }^{36}$, il n'appartient qu'au pays de Tendre d'imaginer tout à la fois une relation inédite entre les sexes, autant fondée sur le mérite - Estime et Reconnaissance - qu'entraînée par la force de l'Inclination, et d'en tenter alors une nouvelle forme d'expression littéraire: accordée aux critères de l'esthétique galante, mais infléchie par cette modulation spécifique du style. En partie héritière de l'ancienne catégorie de la «douceur», celle-ci permet de vérifier, en dernière analyse, le statut de Tendre comme lieu de médiations, cette fois sur le plan esthétique. Le champ des représentations socioculturelles débouche alors sur un ensemble de manifestations stylistiques, qui n'en sont pas simples reflets ni traductions.

C'est en ce point que l'enquête sur les inventions de Tendre doit s'assortir d'études précises consacrées à ses formes d'expression. La belle conversation, l'art périlleux du compliment ou de la déclaration d'amour, la technique du portrait ou de la description, ont déjà permis de mieux comprendre la logique souterraine qui anime cette généreuse mais fragile stylisation du monde, nécessairement silencieuse ou peu diserte sur des défauts dont il revient en revanche au «roman comique» ou aux œuvres satiriques de faire l'inventaire sans concessions.

Car le Tendre est d'abord affaire de discours : paraphrases en prose et variations versifiées (églogues, stances, caprices, dialogues, etc.) en témoignent ${ }^{37}$; aux «Grands Jours» de Sapho, les jugements sont rendus sous cette forme:

36. Comme l'écrit Sapho à Acante: «J'ai à vous avertir qu'en matière d'amitié, je prends les choses au pied de la lettre, et que ce n'est qu'en galanterie que je souffre l'exagération.» (CS, p. 61)

37. Voir les «Annexes» de l'édition citée des Chroniques du Samedi, p. 285-329. 
Quand cette fille sans pareille,

Sapho notre grande merveille,

La mère des tendres discours,

Au jardin tenait ses grands jours,

[...

Chacun y parlait de tendresse,

Lettre, billet, ou compliment,

Tout finissait par tendrement,

De travers ou de bonne grâce

Tendre trouvait par tout sa place $^{38}[\ldots]$

Et le critère de réussite littéraire en est explicite, le Tendre enchérissant sur l'héritage galant:

Les vers que vous m’avez donnés

Sont si galants, si bien tournés,

Qu'ils sont tous faits, je vous le jure,

Comme s'ils étaient de Voiture.

Encore ne sais-je pas bien

Si je ne leur dérobe rien:

Car si je les sais bien entendre,

Ils ont quelque chose de tendre

Que ceux de Voiture n'ont pas,

Qui leur donne certains appas.

(«Sapho à Thrasile», CS, p. 135)

Plusieurs contemporains de Madeleine de Scudéry s'accordèrent à identifier dans sa manière d'écrire cette qualité «tendre» du style, raillée comme «languissante» par ses détracteurs ${ }^{39}$. Repérable en de nombreux postes d'analyse - liaison des phrases et des propositions (le «doux-coulant» naguère vanté

38. La pièce est de Pellisson; ici dans le Recueil La Suze-Pellisson, Lyon, Antoine Boudet, 1695 , t. I, p. 173.

39. «Pour moi quand je vois écrire d'une manière si tendre, je dis qu'elle ne peut pas manquer de persuader l'esprit; puis qu'elle sait si bien toucher le cœur. » (Marguerite Buffet, Nouvelles observations sur la langue française, où il est traité des termes anciens et inusités, et du bel usage des mots nouveaux. Avec les éloges des illustres savantes, tant anciennes que modernes, Paris, Jean Cusson, 1668, p. 246-247) Un siècle plus tard encore, la Bibliothèque universelle des romans qualifie Le grand Cyrus d'"Ouvrage écrit du style le plus mielleux, le plus tendre, le plus amoureux.» (livraison de novembre 1775, p. 87) 
chez un Des Essarts traduisant les Amadis de Gaule), simplicité lexicale rehaussée par les figures du dialogue et de la fiction ${ }^{40}$, fluidité métrique imposant ses cadres clairs à la syntaxe - , celle-ci se déploie en tous les «genres d'écrire » auxquels s'essayèrent les amis de Sapho.

Chansons et élégies s'avéraient tout particulièrement propices à l'expression de cette poétique du Tendre, confiée à des voix féminines. Madeleine de Scudéry elle-même s'y illustra ${ }^{41}$, et surtout la comtesse de La Suze, louée dès 1658 par la romancière. La première édition du Recueil La Suze-Pellisson leur fera une large place, mais sans chercher à les distinguer des autres pièces galantes. Pourtant, le « je ne sais quoi de doux, de languissant, et de passionnét2 » qui constitue le propre caractère de l'élégie française selon les (rares) théoriciens du XVII ${ }^{\mathrm{e}}$ siècle ${ }^{43}$ aurait pu servir à en identifier plus précisément la veine. André Renaud la définit ainsi, à la fin du siècle :

Pour l'Élégie elle ne doit jamais s'exprimer que par le Langage du cœur, autrement il est ridicule qu'elle pleure par art, qu'elle ne parle que de soupirs ardents, lorsque le cœur est tout glacé; elle doit être remplie de tendres sentiments; les passions y doivent être touchées d'une manière tout à fait délicate, surtout la Passion qu'on appelle par excellence passion et la belle passion. Pour cela l'Amour qui se fait le

40. Qui animent les «cycles» poétiques (de la Fauvette, de la Pigeonne, des Poires et de l'Oranger, etc.) ainsi que les dialogues allégoriques de Pellisson ou Isarn : voir Élisa Biancardi, «Madeleine de Scudéry et son cercle: spécificité socioculturelle et créativité littéraire », Papers on French Seventeenth Century Literature, vol. XXII, n 43, 1995, p. 415-429.

41. Voir Renate Kroll, «Femme poète» im Grand Siècle. Zur Lyrik der Madeleine de Scudéry im Kontext der "poésie précieuse », Tübingen, Niemayer, 1996, et pour la chanson, Anne-Madeleine Goulet, "Les divertissements musicaux du Samedi », dans Madeleine de Scudéry: une fermme de lettres au XVII e siècle, p. 203-216.

42. C'est en ces termes que Madeleine de Scudéry compose en 1658 le portrait de $\mathrm{M}^{\mathrm{me}}$ de La Suze, dans la quatrième partie de Clélie. Voir Madeleine de Scudéry, «De l'air galant» et autres Conversations (1653-1684). Pour une étude de l'archive galante, Delphine Denis (éd.), Paris, Éditions Honoré Champion, coll. «Sources classiques», $\mathrm{n}^{\circ} 5,1998$, p. 241.

43. Pour une étude du genre au siècle précédent, voir Christine M. Scollen, The Birth of the Elegy in France: 1500-1550, Genève, Slatkine, coll. "Travaux d'humanisme et Renaissance", 1967. On se reportera aisément, du côté des poètes et théoriciens de la même période, à l'édition procurée par Francis Goyet des Traités de poétique et de rhétorique de la Renaissance, Paris, Libraire Générale Française, coll. «Le livre de poche classique ", 1990. On ne saurait trop regretter ici l'absence d'une pareille synthèse sur l'élégie - et d'abord, de travaux monographiques - pour le XVII siècle français. 
plus sentir dans l'Élégie, ne doit rien avoir d'aveugle, de brutal, de déréglé, ce doit être un amour fidèle, constant et honnête, comme celui que l'on a pour une personne de grande vertu et de grand mérite sans aucune relation à la brutalité et aux plaisirs des $\mathrm{Sens}^{44}$.

Douceur et tendresse du style se verront plus tard détachées de cet ancrage éthique; à peine l'insistance sur le «sentiment» en conserve-t-elle une trace ténue:

À l'égard du style, il doit être doux, naturel, touchant et sentimenté. Mais ceci pour être développé demanderait un trop long détail; car il y a une différence bien fine et bien déliée entre la douceur, le naturel, la tendresse du style qu'exige l'Élégie, et les mêmes qualités de celui qui convient aux pièces d'un autre genre ${ }^{45}$.

De formation précaire, l'univers de Tendre se présente pourtant, au terme de cette brève promenade, comme un essai de médiations aux propositions moins stériles qu'il n'y paraît. Dans l'ordre d'une politique des comportements privés, il aura contribué à séparer de plus en plus nettement ces deux modalités du lien interpersonnel que sont l'amour et l'amitiét ${ }^{46}$, tout en ménageant, à l'intersection de l'un et l'autre, un lieu réservé à l'intimité des cœurs, domaine du sensible qu'on n'appelle pas encore affectivité. On a vu, de la même manière, qu'il n'entendait pas faire sécession d'avec le plus vaste Royaume de Galanterie, aux frontières indécises et mobiles: mais les signes de la sociabilité galante, tout à la fois suspects dans leur interprétation et légitimes dans leur expression

44. André Renaud, Manière de parler la langue française selon ses différents styles; avec la critique de nos plus célèbres écrivains, En prose et en vers; et un petit traité de l'orthographe et de la prononciation française, Lyon, Claude Rey, 1697, p. 308-309. Mais l'auteur exprime aussitôt d'importantes réserves à l'égard de la comtesse de La Suze, dont les indéniables talents élégiaques auraient été mieux inspirés au service de l'amour de Dieu...

45. Michel Mourgues, Traité de la poësie françoise: nouvelle édition revue, corrigée et augmentée avec plusieurs observations sur chaque espèce de poésie, Paris, Jacques Vincent, 1724 [1685], p. 272.

46. La composition, dans la même décennie, du dialogue allégorique de Charles Perrault, Dialogue de l'amour et de l'amitié, Paris, Estienne Loyson, 1660, prend acte de cette confrontation désormais conventionnelle. 
ritualisée, ne sauraient se confondre, en dépit des apparences, avec les manifestations du Tendre; ces dernières invitent en réalité à une complète resémantisation des premiers, à leur pleine réévaluation. Code certes partagé, mais qui ne suscite pas une adhésion de semblable nature. Paradoxalement, si le modèle sentimental du Tendre semble élaboré pour le secret du for intérieur, il ne saurait être opératoire que publié. Les récits d'invention qui l'encadrent espéraient sans doute, tout en procédant à cette divulgation, en conjurer les effets pernicieux par un puissant dispositif de contextualisation: le processus s'en voudrait fortement contraignant, de manière à limiter les errances d' «applications » déplacées, d'interprétations sinon malveillantes, tout au moins aberrantes; mais, actualisé en ses deux «formats » distincts (recueil manuscrit et roman), il préserve du jeu entre une stricte lecture référentielle et les possibles de la fiction narrative.

Conversations intimes de vive voix, échangées pourtant sous le regard de tous, archivage manuscrit en manière d'œuvre collective des jeux du Tendre, enfin publication imprimée livrée à l'ensemble des lecteurs: on aura reconnu là, dans toute leur complexe porosité, trois médiums de communication dont il convient de comprendre les logiques propres. Elles entrelacent sans les mêler événements privés et figurations publiques, témoignages personnels et récits publiables; elles engagent autant les destinataires restreints d'une compagnie choisie qu'un lectorat tout aussi anonyme qu'imprévisible, aléatoire, dont les modes d'appropriation et d'interprétation du texte échappent par nécessité à tout contrôle.

Le royaume de Tendre nous convie ainsi à résister à l'opposition hâtive quoique souvent de bonne méthode! - entre récits de vie et création littéraire. Les Chroniques du Samedi, qui archivèrent la naissance du Tendre, se situent au confluent des deux discours, jusque dans leur hybridité matérielle. L'invention de soi, saisie au plus juste de son dess[e]in - intention de sens et figuration - articule ici la véracité du témoignage autobiographique à la vérité d'un «mensonge romanesque » assumé en conscience. Pas plus que l'un n'explique l'autre, l'œuvre ne s'y épuise en reflet, fût-il idéalisé. De quoi laisser librement s'épanouir la dynamique d'une circulation aussi réglée qu'ouverte, pour qui accepte pareil scrupule de lecture: c'est où, peut-être, une philologie bien entendue pourrait prendre à la lettre l'injonction à mieux aimer... 